BARI-TH 519/05

\title{
Smeared gap equations in crystalline color superconductivity
}

\author{
M. Ruggieri \\ Università di Bari, I-70126 Bari, Italia \\ and \\ I.N.F.N., Sezione di Bari, \\ I-70126 Bari, Italia
}

\begin{abstract}
In the framework of HDET, we discuss an averaging procedure of the NJL quark-quark interaction lagrangian, treated in the mean field approximation, for the two flavor LOFF phase of QCD. This procedure gives results which are valid in domains where Ginzburg-Landau results may be questionable. We compute and compare the free energy for different LOFF crystalline structures.
\end{abstract}




\section{INTRODUCTION AND THE LOFF STATE}

The behaviour of QCD at very high baryon density and low temperature has recently attracted a lot of interest. In these conditions, quarks are expected to deconfine [1] and occupy (in momentum space) large Fermi spheres. At very high densities the relevant interaction is the one gluon exchange, which is attractive in the antisymmetric $\overline{\mathbf{3}}$ color channel. Thus, a Cooper-like pairing phenomenon is expected to occur. This is color superconductivity [2, 3, 4, 5, 6, 7, 8, 9] (see [10, 11, 12, 13, 14] for reviews).

Regimes of high baryon densities and low temperatures are expected to be realized in the core of compact stars; therefore these superdense objects could be the places where color superconductivity is realized in nature. Apart the astrophysical speculations, the study of color-superconductive QCD is an intriguing challenge itself because its understanding implies a deeper understanding of the QCD phase diagram.

In nature, as a consequence of electrical and color neutrality and of the different masses, the Fermi momenta of the quarks should depend on their color and their flavor. When the difference of the Fermi momenta of the pairing quarks are too different, a superconductive ground state in which the Cooper pairs have a net momentum is energetically favored with respect to the usual, zero momentum state. The resulting state is known as LOFF phase, and has been studied in the sixties in the context of condensed matter physics [15, 16]. Possible realizations of the LOFF state in the frame of color superconductivity have been considered for the first time in Refs. [17, 18, 19] (see [20] for a review and Refs. 21, 22, 23] for recent developments).

A color-superconductive phase is characterized by a nonzero expectation value of a bilinear quark operator, namely a condensate. In the LOFF phase with two flavors $(u$ and $d)$ one has

$$
\left\langle\psi_{\alpha i} C \gamma_{5} \psi_{\beta j}\right\rangle \propto \Delta(\boldsymbol{r}) \epsilon^{\alpha \beta 3} \epsilon_{i j 3}
$$

where $\alpha, \beta$ are color indices and $i, j$ denote the flavor. A three flavor case $(u, d$ and $s)$ has been recently considered in Ref. 24]. As for the space dependence of the condensate one usually decomposes $\Delta(\boldsymbol{r})$ as a sum of plane waves. The most simple ansatz is [16]:

$$
\Delta(\boldsymbol{r})=\Delta e^{2 i \mathbf{q} \cdot \mathbf{r}}
$$

and the corresponding superconductive phase is known as FF state. The net momentum 
of the Cooper pair is $\mathbf{2 q}$, which is the same for all the pairs while its direction is chosen spontaneously.

One of the most important problem is to calculate the gap parameter $\Delta$ in Eq. (2). For the FF state this problem can be solved exactly: one has simply to shift the momenta of the paired quarks, $\mathbf{p}_{u} \rightarrow \mathbf{p}_{u}+\mathbf{q}, \mathbf{p}_{d} \rightarrow \mathbf{p}_{d}-\mathbf{q}$ so the $\mathbf{q}$ dependence of the gap parameter disappears. Then one can write a self-consistency and exactly soluble equation for $\Delta$. In the context of QCD this procedure has been applied in Ref. 18] where a diagrammatic approach to the FF color superconductive state is considered. Unfortunately, this procedure cannot be applied to the general case of the linear combination of $N$ plane waves. To overcome this difficulty, one can employ a Ginzburg-Landau (GL) expansion of the general LOFF free energy functional $\Omega$. GL expansion works where $\Delta$ is small when compared to the typical mass scales of the model, in this case $q$ and $\delta \mu$. In the framework of the GL expansion, in

Ref. [19] it is conjectured that a face centered cube is a good candidate for the crystalline color superconductive state.

In this paper we wish to define a procedure that allows to study the crystalline color superconductor with a generic crystalline structure in a space of parameters which is complementary to the GL one. This procedure is based on a weighted average of the interaction lagrangian over the lattice cell. The paper is organized as follows: in Sec. II we discuss the smearing procedure used to obtain the effective gap equations, in the simple case of the FF state. Sec. [II is devoted to the generalization of this procedure to a generic crystalline structure. In Sec. IV we show one result of the solution of the smeared gap equations, namely the free energy plots for the different crystalline structures. Finally, in Sec. $\nabla$ we summarize the leading results of our work.

\section{SMEARED LAGRANGIAN IN THE FF PHASE}

We shall consider Cooper pairing of the massless quarks up $u$ and down $d$, with chemical potential $\mu_{u}, \mu_{d}$. We define $\mu=\left(\mu_{u}+\mu_{d}\right) / 2$ and $\delta \mu=\left|\mu_{u}-\mu_{d}\right| / 2 \ll \mu$. We begin with a review of the FF state. Although this case can be solved exactly, it is useful to consider it here in order to fix the notations and introduce some definitions to be used later on.

We work in the framework of High Density Effective Theory (HDET) 12, 25, 26, 27, 28, 
29, 30]. The lagrangian for free quarks can be written as

$$
\mathcal{L}_{0}=\sum_{\vec{v}}\left[\psi_{+}^{\dagger} i V \cdot \partial \psi_{+}+\psi_{-}^{\dagger} i \tilde{V} \cdot \partial \psi_{-}\right]+(L \rightarrow R)
$$

Here the sum represents an average over velocities; $\psi_{ \pm} \equiv \psi_{ \pm \mathbf{v}}$ are velocity dependent, positive energy left handed fields (the negative energy part has been integrated out). $\psi_{\mathbf{v}}$ depends on the residual momentum $\ell$, corresponding to the decomposition of the quark momentum $p=\mu v+\ell$, with $v^{\mu}=(0, \mathbf{v})$ and $\ell_{\|}=\ell \cdot \mathbf{v}=\xi$. We also introduce $V^{\mu}=(1, \mathbf{v})$ and $\tilde{V}^{\mu}=(1,-\mathbf{v})$.

Next we turn to the interaction term. We consider a Nambu-Jona Lasinio (NJL) inspired four fermion interaction to mimic the one gluon exchange of QCD, namely [17, 31]

$$
\mathcal{L}_{I}=-\frac{3}{8} G \bar{\psi} \gamma^{\mu} \lambda_{a} \psi \bar{\psi} \gamma^{\mu} \lambda_{a} \psi
$$

Here $G$ is a coupling constant, with dimension mass $^{-2} ; \lambda_{a}$ are color matrices and a sum over flavors is understood (the FF state with quark-quark interaction mediated by one gluon exchange has been considered in Ref. [32]). In the mean field approximation, after Fierzing, we get

$$
\mathcal{L}_{I}=-\frac{1}{2} \epsilon_{\alpha \beta 3} \epsilon^{i j}\left(\psi_{i}^{\alpha} \psi_{j}^{\beta} \Delta(\boldsymbol{r})+\text { c.c. }\right)+(L \rightarrow R)-\frac{1}{G} \Delta(\boldsymbol{r}) \Delta^{*}(\boldsymbol{r}),
$$

where $i, j$ are flavor indices and $\alpha, \beta$ are color indices. In the FF state the total momentum of the Cooper pair is $\mathbf{2} \mathbf{q}$ and the condensate has the space-dependence of a single plane wave, see Eq. (21). In the HDET formalism Eq. (15) can be recast in the form

$$
\begin{aligned}
\mathcal{L}_{I}= & -\frac{\Delta}{2} \sum_{\mathbf{v}_{\mathbf{i}}, \mathbf{v}_{\mathbf{j}}} \exp \left\{i \mathbf{r} \cdot \boldsymbol{\alpha}\left(\mathbf{v}_{\mathbf{i}}, \mathbf{v}_{\mathbf{j}}, \mathbf{q}\right)\right\} \epsilon_{\mathbf{i j}} \epsilon_{\alpha \beta \mathbf{3}} \psi_{\mathbf{v}_{\mathbf{i}} ; \mathbf{i} \alpha}^{\mathbf{T}}(\mathbf{x}) \mathbf{C} \psi_{-\mathbf{v}_{\mathbf{j}} ; \mathbf{j} \beta}(\mathbf{x}) \\
& -(L \rightarrow R)+\text { h.c. }-\frac{1}{g} \Delta(\boldsymbol{r}) \Delta^{*}(\boldsymbol{r})
\end{aligned}
$$

where

$$
\boldsymbol{\alpha}\left(\mathbf{v}_{\mathbf{i}}, \mathbf{v}_{\mathbf{j}}, \mathbf{q}\right)=2 \mathbf{q}-\mu_{i} \mathbf{v}_{\mathbf{i}}-\mu_{j} \mathbf{v}_{\mathbf{j}}
$$

Eqs. (3) and (71) are the HDET lagrangian of paired quarks in the FF state. Armed with them one can write a gap equation, namely a self-consistence (Schwinger-Dyson) equation for the gap parameter $\Delta$.

Now we have the ingredients necessary to define our smearing procedure. To this end we recall the exact $\mathrm{FF}$ gap equation [31],

$$
\Delta=i \frac{g \rho}{2} \int \frac{d \mathbf{v}}{4 \pi} \int_{0}^{\delta} \frac{d \xi}{2 \pi} \int d \ell_{0} \frac{\Delta_{e f f}}{\ell_{0}^{2}-\xi^{2}-\Delta_{e f f}^{2}}=\frac{g \rho}{2} \int \frac{d \mathbf{v}}{4 \pi} \int_{0}^{\delta} d \xi \frac{\Delta_{e f f}}{\sqrt{\xi^{2}+\Delta_{e f f}^{2}}}
$$


where we have defined an effective gap parameter,

$$
\Delta_{e f f}=\Delta \theta\left(E_{u}\right) \theta\left(E_{d}\right)= \begin{cases}\Delta & \text { for }(\xi, \mathbf{v}) \in P R \\ 0 & \text { elsewhere }\end{cases}
$$

and $E_{u, d}$ are the dispersion laws for $u$ and $d$ quarks respectively [17, 33

$$
E_{d, u}= \pm \delta \mu \mp \mathbf{q} \cdot \mathbf{v}+\sqrt{\xi^{2}+\Delta^{2}}
$$

$P R$ denotes the pairing region,

$$
P R=\left\{(\xi, \mathbf{v}) \mid E_{u}>0 \text { and } E_{d}>0\right\} .
$$

We stress that Eq. (8) is exact. The key observation is that we can obtain the same gap equation (8) in the framework of HDET by defining a weighted smearing procedure of the gap lagrangian (6) over the lattice cell. First of all, we note that in the gap equation the relevant momenta are small with respect to the gap which is of the order of $q$. Therefore we may assume that the velocity dependent fields are slowly varying over regions of the order of the lattice size. This means that in the average we can treat them as constant, and in conclusion the average is made only on the coefficient $\exp \{i \mathbf{r} \cdot \boldsymbol{\alpha}\}$. Therefore what we are computing is

$$
I(\boldsymbol{\alpha})=\left\langle\exp \{i \mathbf{r} \cdot \boldsymbol{\alpha}\} g_{R}(\mathbf{r})\right\rangle
$$

where the bracket means average over the cell, and the weight function $g_{R}(\mathbf{r})$ can be chosen in such a way that

$$
I(\boldsymbol{\alpha})=\delta_{R}^{3}\left(\frac{\boldsymbol{\alpha}}{2 q}\right), \quad \text { where } \quad \delta_{R}^{3}(\mathbf{x})= \begin{cases}1 & \text { for }|\mathbf{x}|<\frac{\pi}{2 R} \\ 0 & \text { elsewhere }\end{cases}
$$

and $R / \pi \approx 1$. Independently of the exact form of $g_{R}(\mathbf{r})$, we will assume that the average procedure gives as a result the brick-shaped function $\delta_{R}$ defined in (13). As shown in Ref. [31], choosing

$$
R=\frac{\pi|\delta \mu-\mathbf{v} \cdot \mathbf{q}|}{2 \sqrt{\xi^{2}+\Delta^{2}}|h(\mathbf{v} \cdot \hat{\mathbf{q}})|}, \quad h(\mathbf{v} \cdot \hat{\mathbf{q}})=1-\frac{z_{q}}{\mathbf{v} \cdot \hat{\mathbf{q}}}
$$


one obtains from Eq. (6) the smeared lagrangian

$$
\mathcal{L}_{I}=-\frac{1}{2} \sum_{\vec{v}} \Delta_{e f f} \epsilon_{i j} \epsilon_{\alpha \beta 3} \psi_{\mathbf{v} ; i \alpha}^{T}(\ell) C \psi_{-\mathbf{v} ; j \beta}(-\ell)-(L \rightarrow R)+\text { h.c. }-\frac{1}{g} \Delta \Delta^{*}
$$

from which the desired gap equation (8) is obtained (the fermion propagator can be read in Eqs. (22) and (23) of Ref. 31]). Since $R / \pi \approx 1$, then $\Delta$ has not to be small, meaning that we should be far from a second order phase transition.

Let us finally notice that in Eq. (8) one can perform the $\ell_{0}$ integration by substituting the previous expression for $R$ with

$$
R=\frac{\pi|\delta \mu-\mathbf{v} \cdot \mathbf{q}|}{2\left|\ell_{0}\right| \cdot|h(\mathbf{v} \cdot \hat{\mathbf{q}})|}
$$

In fact, observing that in any case $\Delta_{e f f}$ is equal to 0 or $\Delta$, at the pole we get back the expression (14). Then written as in (16), $R$ and analogously $\Delta_{\text {eff }}$ become functions of the velocity and the energy; therefore our average should be better taken in the momentum space.

\section{SMEARING FOR GENERIC CRYSTALLINE STRUCTURES}

We have defined in the previous section a smearing procedure which, in the case of the FF state, allows to write in the HDET formalism a gap equation which coincides with the exact gap equation in Eq. (8) . Apart the technical details for the definition of the weighting function, the smearing procedure can be view as a recipe which allows to replace an $\mathbf{r}$ dependent gap function by an $\mathbf{r}$-independent one. All the details of the crystalline structure are embodied into the definition of the pairing regions.

We now use this recipe to smear the interaction lagrangian for generic crystalline structures, defined by the pairing ansatz

$$
\Delta(\boldsymbol{r})=\Delta \sum_{m=1}^{P} e^{2 i \mathbf{q}_{\mathbf{m}} \cdot \mathbf{r}}, \quad \mathbf{q}_{\mathbf{m}}=q \mathbf{n}_{\mathbf{m}} .
$$

This procedure, although artificial, allows to study generic crystalline structures in a domain where Ginzburg-Landau expansion may not work well. Generalizing the results of the previous equations we substitute in the Lagrangian (15) $\Delta_{\text {eff }}\left(\mathbf{v} \cdot \mathbf{n}, \ell_{0}\right)$ with

$$
\Delta_{E}\left(\mathbf{v}, \ell_{0}\right)=\sum_{m=1}^{P} \Delta_{e f f}\left(\mathbf{v} \cdot \mathbf{n}_{\mathbf{m}}, \ell_{0}\right)
$$


and the arguments of the $\theta$ functions in Eq. (9) will be the appropriate fermion dispersion laws. After smearing the gap equation reads

$$
P \Delta=i \frac{g \rho}{2} \int \frac{d \mathbf{v}}{4 \pi} \int \frac{d^{2} \ell}{2 \pi} \frac{\Delta_{E}\left(\mathbf{v}, \ell_{0}\right)}{\ell_{0}^{2}-\ell_{\|}^{2}-\Delta_{E}^{2}\left(\mathbf{v}, \ell_{0}\right)}
$$

which generalizes Eq. (8). The energy integration is performed by the residue theorem and the phase space is divided into different regions according to the pole positions. We get

$$
P \Delta \ln \frac{2 \delta}{\Delta_{0}}=\sum_{k=1}^{P} \iint_{P_{k}} \frac{d \mathbf{v}}{4 \pi} d \xi \frac{\Delta_{E}(\mathbf{v}, \epsilon)}{\sqrt{\xi^{2}+\Delta_{E}^{2}(\mathbf{v}, \epsilon)}}=\sum_{k=1}^{P} \iint_{P_{k}} \frac{d \mathbf{v}}{4 \pi} d \xi \frac{k \Delta}{\sqrt{\xi^{2}+k^{2} \Delta^{2}}}
$$

where the pairing regions $P_{k}$ are defined as follows

$$
P_{k}=\left\{(\mathbf{v}, \xi) \mid \Delta_{E}(\mathbf{v}, \epsilon)=k \Delta\right\}
$$

and we have ruled out the coupling constant $G$ by means of the BCS gap $\Delta_{0}$. The first term in the sum, corresponding to the region $P_{1}$, has $P$ equal contributions with a dispersion rule equal to the Fulde and Ferrel case. This can be interpreted as a contribution from $P$ non interacting plane waves. In the other regions the different plane waves have an overlap.

Thus for $N$ plane waves the smearing procedure does not simply leads to a lagrangian which is the sum of $N$ plane waves (FF) lagrangians. This "would be nice" scenario is complicated by the presence of the pairing regions with two or more overlaps $\left(P_{2}, \ldots, P_{N}\right)$.

\section{NUMERICAL RESULTS FOR THE FREE ENERGY}

In this section we present results for the free energy $\Omega$ of the structures that we have considered in Ref. 31], computed as integral of the gap equation (20). In particular, in Fig. 1] we show $\Omega(\Delta, \delta \mu)-\Omega(0, \delta \mu)$ against $\delta \mu$ for the face centered (fcc) and the body centered (bcc) cubic structures (the other structures have a higher free energy and therefore they are not shown here). Our central result is that the bcc is a good candidate for the ground state for $\delta \mu \leq 0.95 \Delta_{0}$. Above this value of $\delta \mu$ the fcc is the good ground state for $\delta \mu \leq 1.32 \Delta_{0}$. For higher values of $\delta \mu$, the fermion condensation is energetically disfavored. The transition to the normal state is first order. 


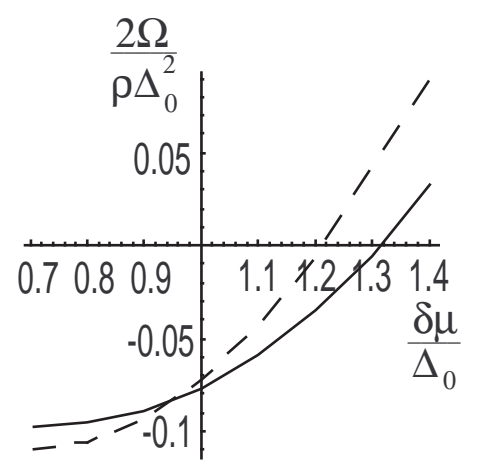

FIG. 1: The values of the free energies of the bcc (dashed line) and of the fcc (full line) crystalline LOFF structures as a function of $\delta \mu / \Delta_{0}$. The bcc is the favored structure up to $\delta \mu \approx .95 \Delta_{0}$; for $.95 \Delta_{0}<\delta \mu<1.32 \Delta_{0}$ the fcc is favored. Here, for each value of $\delta \mu$, the values of $z_{q}$ and $\Delta$ are those that minimize the free energy. From Ref. [31].

\section{CONCLUSIONS}

In the framework of HDET, we have discussed an averaging procedure of the NJL quarkquark interaction lagrangian, treated in the mean field approximation, for the LOFF phase of QCD. This procedure gives results which are valid in domains where Ginzburg-Landau results may be questionable. Among the several structures considered, we find that a body centered cube is the favorite ground state for $\delta \mu \leq 0.95 \Delta_{0}$, while for higher values of $\delta \mu$ the face centered cube is favoured. A first order transition to the normal state is found for $\delta \mu \approx 1.32 \Delta_{0}$. The method exposed here can be applied also to the LOFF phase in the electromagnetic superconductors (see for example Ref. [34]]).

Acknowledgments. I am in debt with R. Casalbuoni, R. Gatto, N. Ippolito, G. Nardulli and M. Ciminale for fruitful collaboration; moreover I thank M. Alford, A. Gerhold, M. Mannarelli and I. Shovkovy for clarifying discussions, comments and helpful correspondence.

[1] J. C. Collins and M. J. Perry, Phys. Rev. Lett. 34, 1353 (1975).

[2] B. C. Barrois, Nucl. Phys. B 129, 390 (1977). 
[3] D. Bailin and A. Love, Phys. Rept. 107, 325 (1984).

[4] M. G. Alford, K. Rajagopal and F. Wilczek, Phys. Lett. B 422, 247 (1998) arXiv:hep-ph/9711395.

[5] R. Rapp, T. Schafer, E. V. Shuryak and M. Velkovsky, Phys. Rev. Lett. 81, 53 (1998) arXiv:hep-ph/9711396.

[6] T. Schafer and F. Wilczek, Phys. Rev. D 60, 114033 (1999) arXiv:hep-ph/9906512.

[7] M. G. Alford, K. Rajagopal and F. Wilczek, Nucl. Phys. B 537, 443 (1999) arXiv:hep-ph/9804403.

[8] I. Shovkovy and M. Huang, Phys. Lett. B 564, 205 (2003) arXiv:hep-ph/0302142.

[9] M. Alford, C. Kouvaris and K. Rajagopal, Phys. Rev. Lett. 92, 222001 (2004) arXiv:hep-ph/0311286.

[10] K. Rajagopal and F. Wilczek, arXiv:hep-ph/0011333.

[11] M. G. Alford, Ann. Rev. Nucl. Part. Sci. 51, 131 (2001) arXiv:hep-ph/0102047.

[12] G. Nardulli, Riv. Nuovo Cim. 25N3, 1 (2002) arXiv:hep-ph/0202037.

[13] T. Schafer, arXiv:hep-ph/0304281.

[14] T. Schaefer, arXiv:hep-ph/0509068.

[15] A. I. Larkin, Yu. N. Ovchinnikov, Zh. Eksp. Teor. Fiz.47, (1964), 1136.

[16] P. Fulde, R. A. Ferrel, Phys. Rev.135 (1964) ,A550.

[17] M. G. Alford, J. A. Bowers and K. Rajagopal, Phys. Rev. D 63, 074016 (2001) arXiv:hep-ph/0008208.

[18] J. A. Bowers, J. Kundu, K. Rajagopal and E. Shuster, Phys. Rev. D 64, 014024 (2001) arXiv:hep-ph/0101067.

[19] J. A. Bowers and K. Rajagopal, Phys. Rev. D 66, 065002 (2002) arXiv:hep-ph/0204079.

[20] R. Casalbuoni and G. Nardulli, Rev. Mod. Phys. 76, 263 (2004) arXiv:hep-ph/0305069.

[21] I. Giannakis and H. C. Ren, Phys. Lett. B 611, 137 (2005) arXiv:hep-ph/0412015.

[22] I. Giannakis, D. f. Hou and H. C. Ren, arXiv:hep-ph/0507306.

[23] I. Giannakis and H. C. Ren, Nucl. Phys. B 723, 255 (2005) arXiv:hep-th/0504053.

[24] R. Casalbuoni, R. Gatto, N. Ippolito, G. Nardulli and M. Ruggieri, arXiv:hep-ph/0507247.

[25] D. K. Hong, Phys. Lett. B 473, 118 (2000) arXiv:hep-ph/9812510.

[26] D. K. Hong, Nucl. Phys. B 582, 451 (2000) arXiv:hep-ph/9905523.

[27] S. R. Beane, P. F. Bedaque and M. J. Savage, Phys. Lett. B 483, 131 (2000) 
arXiv:hep-ph/0002209.

[28] T. Schafer, Nucl. Phys. A 728, 251 (2003) arXiv:hep-ph/0307074.

[29] R. Casalbuoni, AIP Conf. Proc. 602, 358 (2001) arXiv:hep-th/0108195.

[30] T. Schafer, eConf C030614, 038 (2003) arXiv:hep-ph/0310176.

[31] R. Casalbuoni, M. Ciminale, M. Mannarelli, G. Nardulli, M. Ruggieri and R. Gatto, Phys. Rev. D 70, 054004 (2004) arXiv:hep-ph/0404090.

[32] A. K. Leibovich, K. Rajagopal and E. Shuster, Phys. Rev. D 64, 094005 (2001) arXiv:hep-ph/0104073.

[33] R. Casalbuoni, R. Gatto, M. Mannarelli, G. Nardulli, M. Ruggieri and S. Stramaglia, Phys. Lett. B 575, 181 (2003) [Erratum-ibid. B 582, 279 (2004)] arXiv:hep-ph/0307335.

[34] R. Casalbuoni, R. Gatto, M. Mannarelli, G. Nardulli and M. Ruggieri, Phys. Lett. B 600, 48 (2004) arXiv:hep-ph/0407210. 